\title{
Right ventricular free wall longitudinal strain and strain rate quantification with cardiovascular magnetic resonance based tissue tracking
}

\author{
Yang-Yang $\mathrm{Qu}^{1,2}$ (1) $\cdot \mathrm{Hao} \mathrm{Li}^{1} \cdot$ Wolfgang Rottbauer ${ }^{1} \cdot$ Gen-Shan $\mathrm{Ma}^{2,3} \cdot$ Dominik Buckert $^{1} \cdot$ Volker Rasche $^{1}$
}

Received: 15 November 2019 / Accepted: 21 May 2020 / Published online: 27 May 2020

(c) The Author(s) 2020

\begin{abstract}
Cardiovascular magnetic resonance based tissue tracking (CMR-TT) was reported to provide detailed insight into left ventricular mechanical features. However, inadequate knowledge of the right ventricle (RV) mechanical deformation has been acquired by this advanced technique so far. It was the aim of this study to establish reference values of RV free wall (RVFW) global, regional and segmental longitudinal peak strain and strain rate (LS and LSR), and to investigate the gender- and age-related difference as well as the base-to-apex gradient of RVFW-LS and LSR with CMR-TT. 150 healthy volunteers (75 males/females) were retrospectively and continuously recruited and subdivided into three age groups $\left(\mathrm{G}_{20-40}, \mathrm{G}_{41-60}\right.$ and $\mathrm{G}_{61-80}$ ). RVFW global, regional (basal, middle-cavity and apical) and segmental LS (GLS, RLS, SLS) along with systolic and diastolic LSR were generated by post-hoc CMR-TT analysis of standard steady-state free precession long-axis four-chamber view cine images acquired at $1.5 \mathrm{~T}$ field strength. The reference value of myocardial RVFW-GLS was $-24.9 \pm 5.2 \%$. We found that females showed more negative GLS than males except in the youngest group, and no age-related difference of GLS was observed in both gender groups. RLS and SLS presented with the same age-related tendency as GLS. The basal and middle-cavity LS were similar between each other and significantly larger than apical LS. RVFW-GLSR resulted as $1.73 \pm 0.58 \mathrm{~s}^{-1}$ and $1.69 \pm 0.65 \mathrm{~s}^{-1}$ during systolic and diastolic phases, respectively. The diastolic GLSR of males tended to decline with the ageing and was significantly lower than that of females in $\mathrm{G}_{61-80}$ group. Regional and segmental LSR showed significant gender-related differences in certain basal and apical region/segments without any age-related effects. CMR-TT overcomes the difficulty in measuring RV global and segmental deformation. The establishment of the vendor-, gender- and segment-specific reference values of RVFW-LS and LSR is essential for the rapid and efficient utilization of CMR-TT modality in the clinical routine.
\end{abstract}

Keywords Cardiovascular magnetic resonance $\cdot$ Tissue tracking $\cdot$ Right ventricle $\cdot$ Strain $\cdot$ Strain rate

\begin{tabular}{l} 
\\
\hline Volker Rasche and Dominik Buckert shared corresponding \\
authorship. \\
\hline $\begin{array}{l}\text { Dominik Buckert } \\
\text { dominik.buckert@uniklinik-ulm.de }\end{array}$ \\
$\square$ Volker Rasche \\
volker.rasche@uni-ulm.de \\
Yang-Yang Qu \\
quyangyang91@163.com \\
Hao Li \\
hao.li@uni-ulm.de \\
Wolfgang Rottbauer \\
wolfgang.rottbauer@uniklinik-ulm.de
\end{tabular}

$\begin{array}{ll}\text { Abbreviations } \\ \text { ANOVA } & \text { Analysis of variance } \\ \text { AUC } & \text { Area under the curve } \\ \text { BMI } & \text { Body mass index } \\ \text { BSA } & \text { Body surface area }\end{array}$

Gen-Shan Ma

magenshan@hotmail.com

Internal Medicine II, Ulm University Medical Center, Ulm, Germany

2 Medical School of Southeast University, Nanjing, China

3 Department of Cardiology, Zhongda Hospital, Southeast University, Nanjing, China 


$\begin{array}{ll}\text { CI } & \text { Confidence interval } \\ \text { CMR } & \text { Cardiovascular magnetic resonance } \\ \text { CMR-TT } & \begin{array}{l}\text { Cardiovascular magnetic resonance based tis- } \\ \text { sue tracking }\end{array} \\ \text { CoV } & \text { Coefficent of variation } \\ \text { EF } & \text { Ejection fraction } \\ \text { FOV } & \text { Field of view } \\ \text { GLS } & \text { Global longitudinal peak strain } \\ \text { GLSR } & \text { Global longitudinal peak strain rate } \\ \text { HR } & \text { Heart rate } \\ \text { ICC } & \text { Intra-class correlation coefficient } \\ \text { LV } & \text { Left ventricle/left ventricular } \\ \text { r } & \text { Correlation coefficient } \\ \text { RLS } & \text { Regional longitudinal peak strain } \\ \text { RLSR } & \text { Regional longitudinal peak strain rate } \\ \text { RV } & \text { Right ventricle/right ventricular } \\ \text { RVEDVI } & \text { Right ventricular end-diastolic volume index } \\ \text { RVESVI } & \text { Right ventricular end-systolic volume index } \\ \text { RVFW } & \text { Right ventricular free wall } \\ \text { SLS } & \text { Segmental longitudinal peak strain } \\ \text { SLSR } & \text { Segmental longitudinal peak strain rate } \\ \text { SSFP } & \text { Steady-state free precession } \\ \text { SVI } & \text { Stroke volume index } \\ \text { TE } & \text { Echo time } \\ \text { TR } & \text { Repetition time } \\ & \end{array}$

\section{Background}

There is strong evidence that impairment of right ventricular (RV) function plays an important role in developing myocardial diseases [1,2], including cardiomyopathies, and congenital, ischemic, valvular and pulmonary heart diseases [3-5]. The quantification of RV function has increasingly gained clinical interest and relevance over recent years. RV measurements as such are challenging due to its complex anatomical shape, thin trabeculated myocardium, significant load dependence, and variability of filling with respiration.

Cardiovascular magnetic resonance (CMR) has proven promising for providing high spatial and temporal resolution data [6] of the RV without the known limitations of echocardiography introduced by the RV retrosternal position and thin myocardium (3-5 mm) [7, 8]. Traditionally, RV ejection fraction (EF) derived from the volumetric changes of the RV between end-diastole (EDV) and end-systole (ESV) phases is regarded as a powerful and independent parameter to evaluate RV global contractile function, but cannot provide details on global or regional myocardial deformation. Myocardial strain analysis has been reported as a sensitive surrogate to EF to detect subclinical alterations of myocardial function $[9,10]$, with remarkable diagnostic and prognostic value in heart failure, pulmonary hypertension and ischemic heart disease $[5,8,11,12]$.
CMR based tissue tracking (CMR-TT) enables the posthoc offline analysis of dynamic deformation and derives mechanical global and regional parameters including strain and strain rate (SR) over the whole cardiac cycle from conventional cine data [13-16]. Strain analysis has extensively be evaluated for the left ventricle (LV) and reference values have be published for different analysis tools [17, 18]. Application of the technique to the RV is still limited $[19,20]$ and further vendor-specific reference values are required before wide utilization in clinical routine.

It was the objective of this study to investigate genderand age-specific reference values of RV free wall (RVFW) global, regional and segmental transmural longitudinal peak strain (LS) and strain rate (LSR) in 150 healthy subjects.

\section{Methods}

\section{Study population}

CMR data of 150 patients (mean age $49.8 \pm 17.3$ years) not suffering from cardiovascular diseases were included in this retrospective analysis. Inclusion criteria were: above 18 years of age, $\mathrm{LVEF} \geq 55 \%, \mathrm{RVEF} \geq 45 \%$, with no history of cardiovascular or pulmonary diseases or related risk factors (e.g. coronary artery diseases, cardiac arrhythmia, pulmonary dysfunction, hypertension, dyslipidemia, smoking, diabetes mellitus or impaired glucose tolerance).

To investigate gender- and age-related differences of RVFW-LS and LSR, the enrolled subjects were selected equally into gender groups and subdivided into three age groups as $\mathrm{G}_{20-40}$ (age range 20-40 years), $\mathrm{G}_{41-60}$ (age range 41-60 years) and $\mathrm{G}_{61-80}$ (age range $61-80$ years).

This study was approved by the local ethics committee. All participants provided written informed consent.

\section{CMR protocol}

All participants underwent a conventional CMR examination on a clinical $1.5 \mathrm{~T}$ whole-body MRI system (Achieva 1.5T, Philips Healthcare, Best, The Netherlands) at Ulm University Medical Center. All data were acquired with a cardiac 32-channel phased-array receive coil. A stack of short-axis cine images covering the entire left and right ventricle and long-axis cine images (2, 3, 4-chamber) were acquired applying an electrocardiogram-gated steady-state free precession (SSFP) breathhold sequence. Typical acquisition parameters were as: echo time $\mathrm{TE}=1.5 \mathrm{~ms}$, repetition time $\mathrm{TR}=3.0 \mathrm{~ms}$, flip angle $=55^{\circ}$, spatial resolution $=1.7 \mathrm{~mm} \times 1.7 \mathrm{~mm}$, field of view FOV $=360 \mathrm{~mm} \times 325 \mathrm{~mm}$, slice thickness $\mathrm{s}_{\mathrm{D}}=8 \mathrm{~mm}$, no slice gap, and 32 cardiac phases.

Biventricular morphological and functional assessment was performed by two experienced CMR physicians with 
a standard commercial software package (ViewForum ${ }^{\circledR}$, Philips Healthcare, Best, The Netherlands). All RV volumetric parameters were normalized to the body surface area $\left[\right.$ BSA $\left(\mathrm{m}^{2}\right)=0.007184 \times$ height $^{0.725}(\mathrm{~cm}) \times$ weight $\left.^{0.425}(\mathrm{~kg})\right]$ to minimize the impact of body size. RV end-diastolic volume index (RVEDVI), RV end-systolic volume index (RVESVI) and RV stroke volume index (RVSVI) and RVEF were assessed to quantify RV morphologic and functional properties. DICOM images were exported to an external workstation for subsequent CMR-TT analysis.

\section{CMR-TT analysis}

RVFW-LS and LSR were derived from the RV long-axis four-chamber view using the commercial specialized software $\mathrm{CVI}^{42}$ (version 5.6.3, Circle, Calgary, Canada) [21, 22] by an experienced user of the package.

For segmentation, the RV tricuspid annular plane and apex were marked, followed by the manual delineation of the RV endocardial border and FW epicardial border at end diastole (Fig. 1a). Subsequently, the software automatically propagated and traced myocardial features phase to phase throughout the whole cardiac cycle. In cases of inaccurate tracking of the RV border, manual corrections of delineation were performed.

From the tracked tissue features, $\mathrm{CVI}^{42}$ was used to generate dynamic mechanical information of global and segmental two-dimensional RV strain for all cardiac phases. Global as well as segmental (6 segments with the same length of RVFW at end diastole) LS and LSR were computed via the Lagrangian formulas as: $L S_{t}=\left(L_{t}-L_{0}\right) / L_{0}, L S R_{t}=d L S_{t} / d t$, respectively [13]. $L_{t}$ refers to the final length of the cardiac tissue, and $L_{0}$ represents the initial length at end diastole. Peak values as well as LS and LSR curves of the global myocardium (GLS and GLSR) and segments (SLS and SLSR) were generated. The peak value of time-strain and time-SR curves (Fig. 1d, e) corresponded with the data generated automatically in the text report. Regional LS and LSR were calculated by averaging the respective two segmental values within the corresponding region.

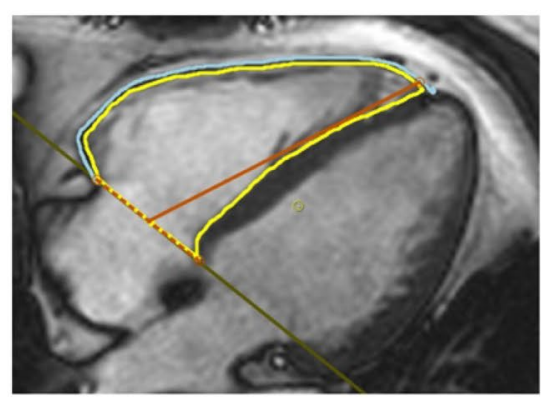

(a)

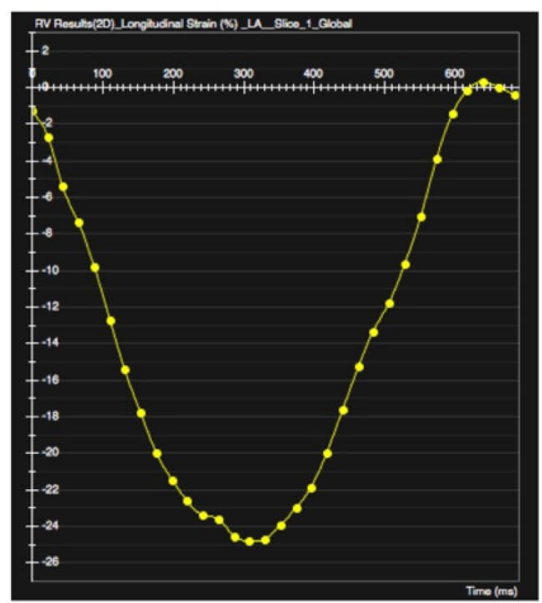

(d)

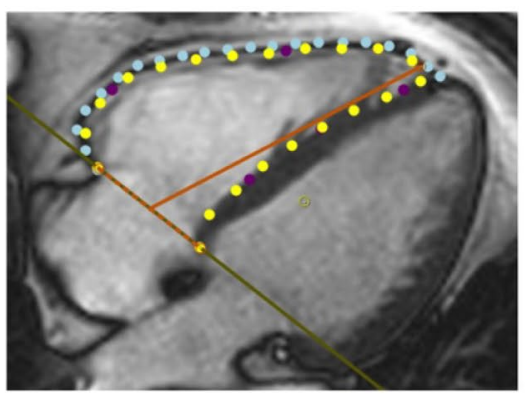

(b)

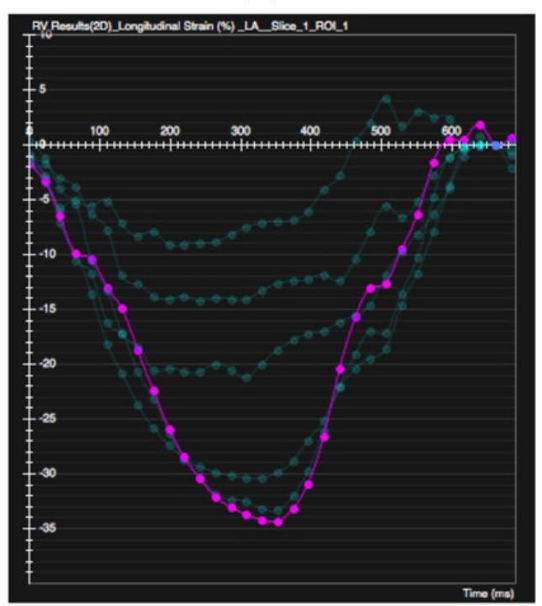

(e)

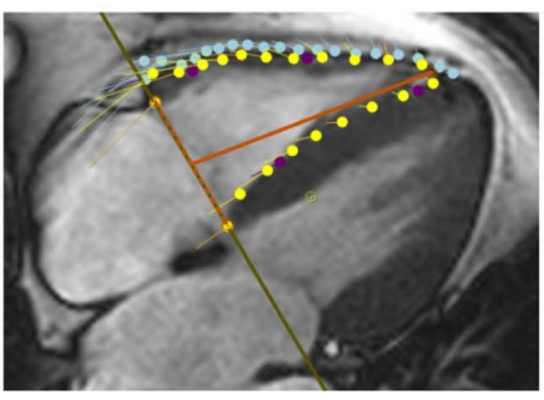

(c)
Fig. 1 CMR-TT analysis and RVFW time-LS curves. a Mark tricuspid annular plane and apex with an orange $\mathrm{T}$ bar, and delineate RV endocardium and RVFW epicardium with yellow and blue lines at end diastole. b The yellow endocardial and blue epicardial boundary points are presented at the phase next to end diastole. $\mathbf{c}$ The posi- tions of boundary points are shown at end systole. The yellow or blue round points represent the final positions of myocardial feature patterns, and the tiny starting points of connected lines indicate the initial positions of feature patterns at end diastole. $\mathbf{d}$ The time-strain curve of RVFW-GLS. e The time-strain curves of RVFW-SLS 


\section{Statistical analysis}

Continuous variables are expressed as mean value with standard deviation (SD). Gaussian distribution was tested applying a Shapiro-Wilk test, and extreme outliers (0/150 to 9/150 for each parameter) were excluded. The statistical significance between two groups or parameters were assessed with a two-tailed Student's $t$ test or a Mann-Whitney $U$ test, as appropriate. Age-related difference among three groups was investigated using analysis of variance (ANOVA) or Kruskal-Wallis test followed by Bonferroni correction, as applicable. Correlation between age and RVFW-GLS was analyzed with a Spearman correlation coefficient (r). Comparisons among basal, middle-cavity and apical LS or LSR were tested with Friedman test. Statistical significance was assumed with $P<0.05$. Intra- and inter-observer variability of RVFW global, regional and segment LS and LSR were performed for fifteen randomly selected subjects using Bland-Altman analysis. Intra-class correlation coefficient (ICC) and coefficient of variation (CoV) were used to assess the reproducibility and variability. All analyses were performed with IBM SPSS 24.0 and Medcalc 19.1.

\section{Results}

\section{Baseline characteristics of the study participants}

The detailed baseline characteristics of the subgroups are presented in Table 1. Average age resulted as $49.8 \pm 17.3$ years old, without a significant difference between males and females in each age group. Compared with females, males showed significantly larger BSA,
RVEDVI, RVESVI and RVSVI, as well as lower RVEF $(P<0.05)$. This tendency got more and more pronounced with increasing age. In the $\mathrm{G}_{61-80}$ group, RVEF of females resulted $12.9 \%$ higher than in males $(69.2 \pm 6.3 \%$ vs. $61.3 \pm 5.6 \%, P<0.05)$. Furthermore, age-related decline of heart rate (HR) was detected in males, and decrease of $\mathrm{RV}$ morphological characters as well as increase of RVEF were observed in females. For females in the $\mathrm{G}_{61-80}$ group, RVEDVI reduced by $24.0 \%$ in comparison with the $\mathrm{G}_{20-40}$ group $\left(61.6 \pm 9.4 \mathrm{~mL} / \mathrm{m}^{2}\right.$ vs. $\left.81.0 \pm 12.4 \mathrm{~mL} / \mathrm{m}^{2}, P<0.05\right)$.

\section{RVFW global, regional and segmental LS}

RVFW global, regional and segmental LS (GLS, RLS and SLS) derived from CMR-TT are provided in Table 2. The reference value of GLS was $-24.9 \pm 5.2 \%$. Females showed higher amplitude of GLS than males in the groups $\mathrm{G}_{41-60}$ and $\mathrm{G}_{61-80}(P<0.05)$. With the increase of age, males presented with smaller GLS, which was opposite to females. However, no significant age-related difference of GLS was noticed in both gender groups $(P>0.05)$ and no correlation was observed between age and GLS $(\mathrm{r}=-0.031, P=0.703$, Fig. 2).

Among the whole population, the LS at middle-cavity and apical regions and segments presented gender-related but no age-related differences. A base-to-apex gradient of RLS was observed. The basal and middle-cavity LS were similar $(P>0.05)$ but significantly larger than apical LS $(P<0.05)$.

\section{RVFW global, regional and segmental LSR}

CMR-TT allows the measurement of RVFW global, regional and segmental LSR (GLSR, RLSR and SLSR) throughout

Table 1 Baseline characteristics of the healthy volunteers

\begin{tabular}{|c|c|c|c|c|c|c|c|c|c|}
\hline & \multicolumn{2}{|l|}{$\mathrm{G}_{20-40}$} & \multicolumn{2}{|l|}{$\mathrm{G}_{41-60}$} & \multicolumn{2}{|l|}{$\mathrm{G}_{61-80}$} & \multirow{2}{*}{$\begin{array}{l}\text { Males } \\
(\mathrm{n}=75)\end{array}$} & \multirow{2}{*}{$\begin{array}{l}\text { Females } \\
(\mathrm{n}=75)\end{array}$} & \multirow{2}{*}{$\begin{array}{l}\text { All } \\
(n=150)\end{array}$} \\
\hline & Males & Females & Males & Females & Males & Females & & & \\
\hline \multicolumn{10}{|l|}{ Baseline demographics } \\
\hline Age (years) & $29.5 \pm 5.5$ & $29.4 \pm 7.4$ & $50.8 \pm 5.7$ & $51.2 \pm 6.5$ & $69.3 \pm 6.1$ & $68.8 \pm 5.7$ & $49.9 \pm 17.3^{\dagger}$ & $49.8 \pm 17.5^{\dagger}$ & $49.8 \pm 17.3$ \\
\hline $\operatorname{BSA}\left(\mathrm{m}^{2}\right)$ & $2.0 \pm 0.2$ & $1.7 \pm 0.1^{*}$ & $2.1 \pm 0.2$ & $1.9 \pm 0.2^{*}$ & $2.0 \pm 0.2$ & $1.8 \pm 0.1^{*}$ & $2.0 \pm 0.2$ & $1.8 \pm 0.2 *^{\dagger}$ & $1.9 \pm 0.2$ \\
\hline $\mathrm{BMI}\left(\mathrm{kg} / \mathrm{m}^{2}\right)$ & $24.9 \pm 3.6$ & $23.8 \pm 6.6^{*}$ & $26.4 \pm 3.9$ & $28.1 \pm 5.9$ & $25.6 \pm 3.8$ & $25.7 \pm 4.5$ & $25.6 \pm 3.8$ & $25.8 \pm 5.9^{\dagger}$ & $25.7 \pm 4.9$ \\
\hline HR (bpm) & $74.0 \pm 18.7$ & $71.6 \pm 11.5$ & $66.3 \pm 16.0$ & $70.9 \pm 14.6$ & $63.8 \pm 14.1$ & $68.6 \pm 11.2$ & $68.0 \pm 16.7^{\dagger}$ & $70.4 \pm 12.4$ & $69.2 \pm 14.7$ \\
\hline \multicolumn{10}{|c|}{ RV morphology and contractile function } \\
\hline $\operatorname{RVEDVI}\left(\mathrm{mL} / \mathrm{m}^{2}\right)$ & $88.9 \pm 16.0$ & $81.0 \pm 12.4$ & $83.1 \pm 17.5$ & $72.7 \pm 11.2 *$ & $78.9 \pm 12.5$ & $61.6 \pm 9.4^{*}$ & $83.6 \pm 15.8$ & $71.8 \pm 13.6^{* \dagger}$ & $77.7 \pm 15.8$ \\
\hline $\operatorname{RVESVI~(mL/m²)~}$ & $38.5 \pm 9.9$ & $31.1 \pm 6.3^{*}$ & $31.8 \pm 10.5$ & $25.3 \pm 7.7 *$ & $30.9 \pm 8.0$ & $20.4 \pm 4.5^{*}$ & $33.7 \pm 10.0^{\dagger}$ & $25.8 \pm 7.6^{* \dagger}$ & $29.7 \pm 9.7$ \\
\hline $\operatorname{RVSVI}\left(\mathrm{mL} / \mathrm{m}^{2}\right)$ & $51.1 \pm 8.9$ & $49.9 \pm 7.9$ & $51.3 \pm 9.1$ & $47.4 \pm 6.8$ & $48.2 \pm 7.0$ & $42.2 \pm 5.1^{*}$ & $50.2 \pm 8.4$ & $46.5 \pm 7.4^{* \dagger}$ & $48.4 \pm 8.1$ \\
\hline $\operatorname{RVEF}(\%)$ & $58.6 \pm 8.0$ & $61.7 \pm 4.1 *$ & $62.5 \pm 6.5$ & $65.6 \pm 7.0$ & $61.3 \pm 5.6$ & $69.2 \pm 6.3^{*}$ & $60.8 \pm 6.9$ & $65.5 \pm 6.6^{* \dagger}$ & $63.2 \pm 7.2$ \\
\hline
\end{tabular}

Results are reported as Mean $\pm \mathrm{SD}$

$B S A$ body surface area, $B M I$ body mass index, $H R$ heart rate, RVEDVI right ventricular end-diastolic volume index, RVESVI right ventricular end-systolic volume index, RVSVI right ventricular stroke volume index, $R V E F$ right ventricular ejection fraction

$* P<0.05$ : males vs. females. ${ }^{\dagger} P<0.05$ : age-related difference among three age groups 
Table 2 RVFW-GLS, RLS and SLS

\begin{tabular}{|c|c|c|c|c|c|c|c|c|c|}
\hline & \multicolumn{2}{|l|}{$\mathrm{G}_{20-40}$} & \multicolumn{2}{|l|}{$\mathrm{G}_{41-60}$} & \multicolumn{2}{|l|}{$\mathrm{G}_{61-80}$} & \multirow{2}{*}{$\begin{array}{l}\text { Male } \\
(\mathrm{n}=75)\end{array}$} & \multirow{2}{*}{$\begin{array}{l}\text { Female } \\
(n=75)\end{array}$} & \multirow{2}{*}{$\begin{array}{l}\text { All } \\
(n=150)\end{array}$} \\
\hline & Males & Females & Males & Females & Males & Females & & & \\
\hline $\begin{array}{l}\text { RVFW-GLS } \\
(\%)\end{array}$ & $24.8 \pm 5.2$ & $-24.5 \pm 5.4$ & $23.3 \pm 4.7$ & $-26.5 \pm 5.2 *$ & $-22.8 \pm 4.9$ & $-27.2 \pm 4.7^{*}$ & $-23.7 \pm 4.9$ & $-26.1 \pm 5.2 *$ & $-24.9 \pm 5.2$ \\
\hline \multicolumn{10}{|c|}{ RVFW-RLS (\%) } \\
\hline Basal & $-28.1 \pm 7.4$ & $-25.9 \pm 9.8$ & $-27.9 \pm 5.9$ & $-27.4 \pm 6.1$ & $-24.6 \pm 6.6$ & $-29.1 \pm 6.2 *$ & $-26.8 \pm 6.8$ & $-27.5 \pm 7.6$ & $-27.1 \pm 7.2$ \\
\hline $\begin{array}{l}\text { Middle- } \\
\text { cavity }\end{array}$ & $-26.2 \pm 5.2$ & $-26.0 \pm 5.7$ & $-25.8 \pm 4.0$ & $-28.9 \pm 4.0^{*}$ & $-26.5 \pm 5.1$ & $-29.5 \pm 4.0^{*}$ & $-26.2 \pm 4.8$ & $-28.1 \pm 4.8^{*}$ & $-27.2 \pm 4.9$ \\
\hline Apical & $-18.1 \pm 5.4$ & $-22.8 \pm 7.1 *$ & $-17.8 \pm 5.4$ & $-21.8 \pm 8.5$ & $-17.3 \pm 5.4$ & $-21.2 \pm 5.8^{*}$ & $-17.8 \pm 5.4$ & $-21.9 \pm 7.1 *$ & $-19.8 \pm 6$ \\
\hline \multicolumn{10}{|c|}{ RVFW-SLS (\%) } \\
\hline Segment 1 & $-27.2 \pm 8.7$ & $-24.5 \pm 12.9$ & $-27.2 \pm 7.6$ & $-26.9 \pm 7.2$ & $-23.0 \pm 7.7$ & $-28.4 \pm 8.4^{*}$ & $-25.8 \pm 8.2$ & $-26.6 \pm 9.8$ & $-26.2 \pm 9.0$ \\
\hline Segment 2 & $-29.0 \pm 6.7$ & $-27.3 \pm 8.5$ & $-28.5 \pm 5.3$ & $-27.8 \pm 6.1$ & $-26.2 \pm 6.5$ & $-29.7 \pm 4.7$ & $-27.9 \pm 6.2$ & $-28.3 \pm 6.6$ & $-28.1 \pm 6.4$ \\
\hline Segment 3 & $-27.5 \pm 6.0$ & $-27.0 \pm 6.4$ & $-28.0 \pm 4.5$ & $-29.2 \pm 5.6$ & $-27.2 \pm 6.2$ & $-30.7 \pm 4.5^{*}$ & $-27.6 \pm 5.5$ & $-29.0 \pm 5.7 *$ & $-28.3 \pm 5.6$ \\
\hline Segment 4 & $-24.9 \pm 5.6$ & $-25.1 \pm 6.8$ & $-23.6 \pm 5.2$ & $-28.5 \pm 4.2 *$ & $-25.1 \pm 6.0$ & $-28.3 \pm 4.2$ & $-24.5 \pm 5.5$ & $-27.3 \pm 5.4^{*}$ & $-25.9 \pm 5.6$ \\
\hline Segment 5 & $-22.7 \pm 5.1$ & $-24.7 \pm 6.0$ & $-21.4 \pm 4.5$ & $-25.2 \pm 6.7^{*}$ & $-20.3 \pm 5.7$ & $-24.9 \pm 5.3 *$ & $-21.5 \pm 5.1$ & $-24.9 \pm 5.9 *$ & $-23.2 \pm 5.8$ \\
\hline Segment 6 & $-13.6 \pm 7.3$ & $-20.9 \pm 10.1^{*}$ & $-14.3 \pm 8.6$ & $-18.4 \pm 11.3$ & $-14.3 \pm 5.9$ & $-17.5 \pm 7.7$ & $-14.1 \pm 7.2$ & $-18.9 \pm 9.8^{*}$ & $-16.5 \pm 8.9$ \\
\hline
\end{tabular}

Results are reported as Mean \pm SD

$R V F W$ right ventricular free wall, $G L S$ global longitudinal peak strain, $R L S$ regional longitudinal peak strain, $S L S$ segmental longitudinal peak strain

$* P<0.05$ : males vs. females
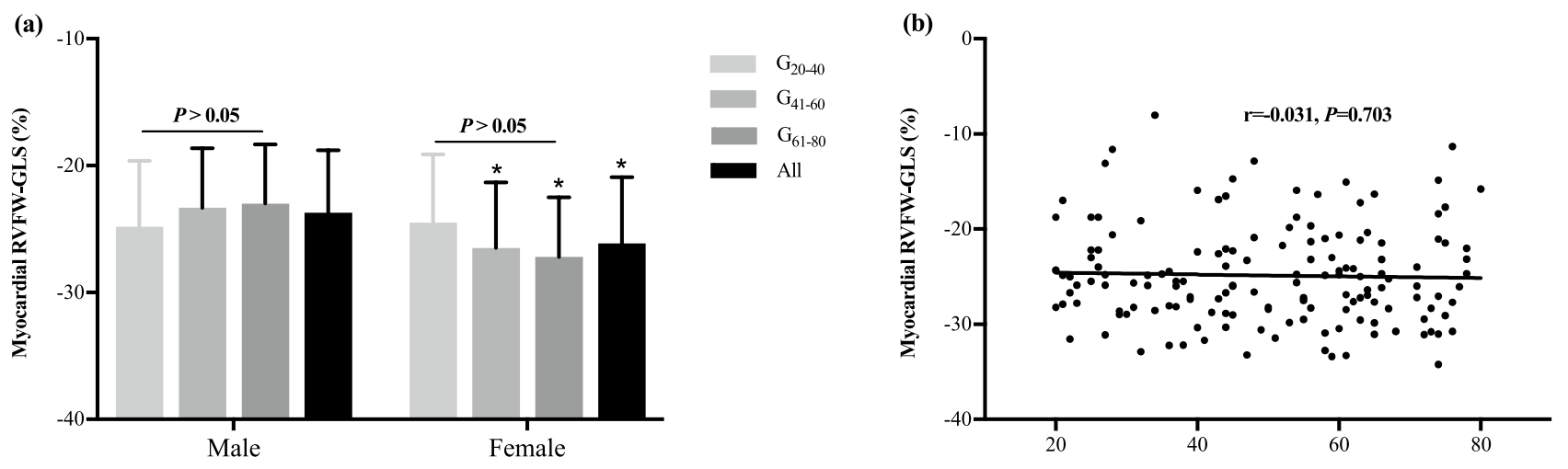

Fig. 2 The reference values of RVFW-GLS and its gender- and agerelated differences. a Gender- and age-related difference of RVFWGLS. Gender-related difference of RVFW-GLS was found in $\mathrm{G}_{41-60}$ and $\mathrm{G}_{61-80}$ groups, and age-associated difference wasn't detected in each gender subgroup. ${ }^{*} P<0.05$ : significant difference between

the whole cardiac cycle, the mean and SD of systolic and diastolic peak SR are presented in Tables 3 and 4.

The systolic GLSR was $-1.68 \pm 0.56 \mathrm{~s}^{-1}$ for males and $-1.78 \pm 0.59 \mathrm{~s}^{-1}$ for females $(P>0.05)$. No significant genderrelated difference of systolic RLSR and SLSR was observed. No significant age-related difference was found for all LSR at systole. Unlike systolic GLSR, diastolic GLSR of males was significantly lower than that of females as $1.59 \pm 0.62 \mathrm{~s}^{-1}$ vs. $1.80 \pm 0.67 \mathrm{~s}^{-1}(P<0.05)$. Similar to diastolic GLSR, a significant gender-related difference of diastolic SLSR was observed in the segment 5 . With the increase of age, diastolic males and females in the same age subgroup. b The correlation between age and RVFW-GLS. There was no correlation between age and RVFW-GLS since Spearman correlation coefficient ( $r$ ) resulted as $-0.031(P=0.703)$

GLSR significantly decreased in males, which is opposite to the trend observed in females.

A base-to-apex gradient of both systolic and diastolic RLS was also observed, which was characterized with the largest RLS value in the base and the smallest RLS value in the apex $(P<0.05)$. 
Table 3 RVFW systolic GLSR, RLSR and SLSR

\begin{tabular}{|c|c|c|c|c|c|c|c|c|c|}
\hline & \multicolumn{2}{|l|}{$\mathrm{G}_{20-40}$} & \multicolumn{2}{|l|}{$\mathrm{G}_{41-60}$} & \multicolumn{2}{|l|}{$\mathrm{G}_{61-80}$} & \multirow{2}{*}{$\begin{array}{l}\text { Male } \\
(n=75)\end{array}$} & \multirow{2}{*}{$\begin{array}{l}\text { Female } \\
(n=75)\end{array}$} & \multirow{2}{*}{$\begin{array}{l}\text { All } \\
(n=150)\end{array}$} \\
\hline & Males & Females & Males & Females & Males & Females & & & \\
\hline $\begin{array}{l}\text { RVFW- } \\
\text { GLSRs } \\
\left(\mathrm{s}^{-1}\right)\end{array}$ & $-1.67 \pm 0.67$ & $-1.57 \pm 0.54$ & $-1.63 \pm 0.43$ & $-1.89 \pm 0.60$ & $-1.73 \pm 0.57$ & $-1.88 \pm 0.58$ & $-1.68 \pm 0.56$ & $-1.78 \pm$ & $9-1.73 \pm 0.58$ \\
\hline \multicolumn{10}{|c|}{ RVFW-RLSRs $\left(\mathrm{s}^{-1}\right)$} \\
\hline Basal & $-2.86 \pm 0.91$ & $-2.44 \pm 0.84$ & $-3.00 \pm 1.01$ & $-2.86 \pm 1.01$ & $-3.00 \pm 1.07$ & $-2.88 \pm 0.85$ & $-2.95 \pm 0.99$ & $-2.73 \pm$ & $1-2.84 \pm 0.95$ \\
\hline $\begin{array}{c}\text { Middle- } \\
\text { cavity }\end{array}$ & $-2.78 \pm 0.98$ & $-2.59 \pm 0.80$ & $-2.56 \pm 0.57$ & $-2.92 \pm 0.89$ & $-2.81 \pm 1.04$ & $-3.04 \pm 1.04$ & $-2.72 \pm 0.88$ & $-2.85 \pm$ & $2-2.79 \pm 0.90$ \\
\hline Apical & $-2.00 \pm 0.61$ & $-2.27 \pm 0.72$ & $-2.08 \pm 0.85$ & $-2.17 \pm 0.64$ & $-1.92 \pm 0.64$ & $-2.06 \pm 0.74$ & $-2.00 \pm 0.70$ & $-2.16 \pm$ & $9-2.08 \pm 0.70$ \\
\hline \multicolumn{10}{|c|}{ RVFW-SLSRs $\left(\mathrm{s}^{-1}\right)$} \\
\hline Segment 1 & $-3.30 \pm 1.64$ & $-2.37 \pm 1.18$ & $-3.17 \pm 1.39$ & $-2.99 \pm 1.33$ & $-3.26 \pm 1.69$ & $-3.10 \pm 1.21$ & $-3.24 \pm 1.56$ & $-2.82 \pm$ & $6-3.03 \pm 1.43$ \\
\hline Segment 2 & $-2.75 \pm 1.18$ & $-2.51 \pm 0.85$ & $-2.85 \pm 0.87$ & $-2.79 \pm 1.00$ & $-2.77 \pm 0.85$ & $-2.67 \pm 0.85$ & $-2.79 \pm 0.97$ & $-2.66 \pm$ & $0-2.72 \pm 0.93$ \\
\hline Segment 3 & $-2.81 \pm 1.04$ & $-2.59 \pm 0.84$ & $-2.69 \pm 0.90$ & $-2.90 \pm 1.07$ & $-3.04 \pm 1.35$ & $-3.26 \pm 1.27$ & $-2.84 \pm 1.11$ & $-2.92 \pm$ & $9-2.88 \pm 1.10$ \\
\hline Segment 4 & $-2.76 \pm 1.09$ & $-2.60 \pm 0.99$ & $-2.44 \pm 0.79$ & $-2.93 \pm 1.02$ & $-2.74 \pm 1.11$ & $-2.83 \pm 0.92$ & $-2.64 \pm 1.00$ & $-2.79 \pm$ & $8-2.71 \pm 0.99$ \\
\hline Segment 5 & $-2.56 \pm 1.11$ & $-2.60 \pm 1.00$ & $-2.61 \pm 1.17$ & $-2.43 \pm 0.72$ & $-2.34 \pm 0.93$ & $-2.36 \pm 0.88$ & $-2.50 \pm 1.06$ & $-2.46 \pm$ & $7-2.48 \pm 0.97$ \\
\hline Segment 6 & $-1.75 \pm 1.01$ & $-1.99 \pm 0.85$ & $-1.62 \pm 0.95$ & $-2.00 \pm 0.98$ & $-1.51 \pm 0.67$ & $-1.79 \pm 0.92$ & $-1.63 \pm 0.88$ & $-1.92 \pm$ & $1-1.77 \pm 0.91$ \\
\hline
\end{tabular}

Results are reported as Mean \pm SD

$R V F W$ right ventricular free wall, GLSRs global longitudinal systolic peak strain rate, RLSRs regional longitudinal systolic peak strain rate, SLSRs segmental longitudinal systolic peak strain rate

Table 4 RVFW diastolic GLSR, RLSR and SLSR

\begin{tabular}{|c|c|c|c|c|c|c|c|c|c|}
\hline & \multicolumn{2}{|l|}{$\mathrm{G}_{20-40}$} & \multicolumn{2}{|l|}{$\mathrm{G}_{41-60}$} & \multicolumn{2}{|l|}{$\mathrm{G}_{61-80}$} & \multirow{2}{*}{$\begin{array}{l}\text { Male } \\
(n=75)\end{array}$} & \multirow{2}{*}{$\begin{array}{l}\text { Female } \\
(n=75)\end{array}$} & \multirow{2}{*}{$\begin{array}{l}\text { All } \\
(n=150)\end{array}$} \\
\hline & Males & Females & Males & Females & Males & Females & & & \\
\hline RVFW-GLSRd $\left(\mathrm{s}^{-1}\right)$ & $1.81 \pm 0.61$ & $1.67 \pm 0.55$ & $1.50 \pm 0.59$ & $1.80 \pm 0.72$ & $1.46 \pm 0.61$ & $1.91 \pm 0.73^{*}$ & $1.59 \pm 0.62^{\dagger}$ & $1.80 \pm 0.67 *$ & $1.69 \pm 0.65$ \\
\hline \multicolumn{10}{|l|}{ RVFW-RLSRd $\left(\mathrm{s}^{-1}\right)$} \\
\hline Basal & $3.10 \pm 1.14$ & $2.53 \pm 1.03$ & $3.16 \pm 1.39$ & $2.96 \pm 1.20$ & $3.13 \pm 1.21$ & $3.23 \pm 0.91$ & $3.13 \pm 1.23$ & $2.91 \pm 1.08$ & $3.14 \pm 1.37$ \\
\hline Middle-cavity & $2.38 \pm 0.90$ & $2.54 \pm 0.94$ & $2.35 \pm 0.85$ & $2.58 \pm 0.82$ & $2.46 \pm 0.99$ & $2.41 \pm 0.66$ & $2.40 \pm 0.90$ & $2.51 \pm 0.81$ & $2.51 \pm 0.97$ \\
\hline Apical & $2.03 \pm 0.79$ & $2.21 \pm 0.82$ & $1.90 \pm 0.75$ & $2.10 \pm 0.69$ & $1.84 \pm 0.70$ & $2.05 \pm 0.79$ & $1.92 \pm 0.74$ & $2.12 \pm 0.76$ & $2.16 \pm 0.99$ \\
\hline \multicolumn{10}{|l|}{ RVFW-SLSRd $\left(\mathrm{s}^{-1}\right)$} \\
\hline Segment 1 & $3.90 \pm 1.99$ & $2.81 \pm 1.61$ & $3.82 \pm 2.04$ & $3.78 \pm 2.37$ & $3.88 \pm 1.96$ & $3.77 \pm 1.45$ & $3.87 \pm 1.97$ & $3.46 \pm 1.89$ & $3.66 \pm 1.93$ \\
\hline Segment 2 & $2.43 \pm 0.59$ & $2.35 \pm 0.96$ & $2.51 \pm 1.11$ & $2.39 \pm 0.88$ & $2.45 \pm 0.90$ & $2.69 \pm 0.95$ & $2.46 \pm 0.88$ & $2.48 \pm 0.93$ & $2.47 \pm 0.90$ \\
\hline Segment 3 & $2.53 \pm 1.03$ & $2.58 \pm 1.22$ & $2.30 \pm 1.11$ & $2.53 \pm 0.93$ & $2.34 \pm 0.90$ & $2.49 \pm 0.73$ & $2.39 \pm 1.01$ & $2.54 \pm 0.97$ & $2.46 \pm 0.99$ \\
\hline Segment 4 & $2.35 \pm 1.07$ & $2.50 \pm 0.87$ & $2.40 \pm 1.04$ & $2.64 \pm 1.04$ & $2.58 \pm 1.22$ & $2.38 \pm 0.82$ & $2.45 \pm 1.10$ & $2.51 \pm 0.91$ & $2.48 \pm 1.00$ \\
\hline Segment 5 & $2.10 \pm 0.96$ & $2.46 \pm 0.76$ & $2.28 \pm 1.10$ & $2.60 \pm 0.96$ & $2.09 \pm 0.92$ & $2.44 \pm 0.80$ & $2.16 \pm 0.99$ & $2.50 \pm 0.84^{*}$ & $2.33 \pm 0.93$ \\
\hline Segment 6 & $1.95 \pm 1.00$ & $2.02 \pm 1.07$ & $1.51 \pm 0.78$ & $1.84 \pm 1.00$ & $1.60 \pm 0.66$ & $1.73 \pm 1.00$ & $1.69 \pm 0.84$ & $1.86 \pm 1.01$ & $1.77 \pm 0.92$ \\
\hline
\end{tabular}

Results are reported as Mean \pm SD

$R V F W$ right ventricular free wall, GLSRd global longitudinal diastolic peak strain rate, $R L S R d$ regional longitudinal diastolic peak strain rate, SLSRd segmental longitudinal diastolic peak strain rate

$* P<0.05$ : males vs. females

${ }^{\dagger} P<0.05$ : age-related difference among three age groups

\section{Intra- and inter-observer reproducibility of RVFW-LS and LSR}

The intra- and inter-observer variability of RVFW global, regional and segmental LS and LSR are presented in Fig. 3 and Tables 5, 6 and 7. Bland-Altman analysis showed that the global evaluation of RVFW-LS revealed the best intraand inter-observer reproducibility $(\mathrm{CoV} \leq 5.11 \%)$ compared with that of systolic and diastolic RVFW-GLSR (CoV ranging from $9.54 \%$ to $16.99 \%$ ). The $\mathrm{CoV}$ of regional and 


\section{Intra-observer variability}
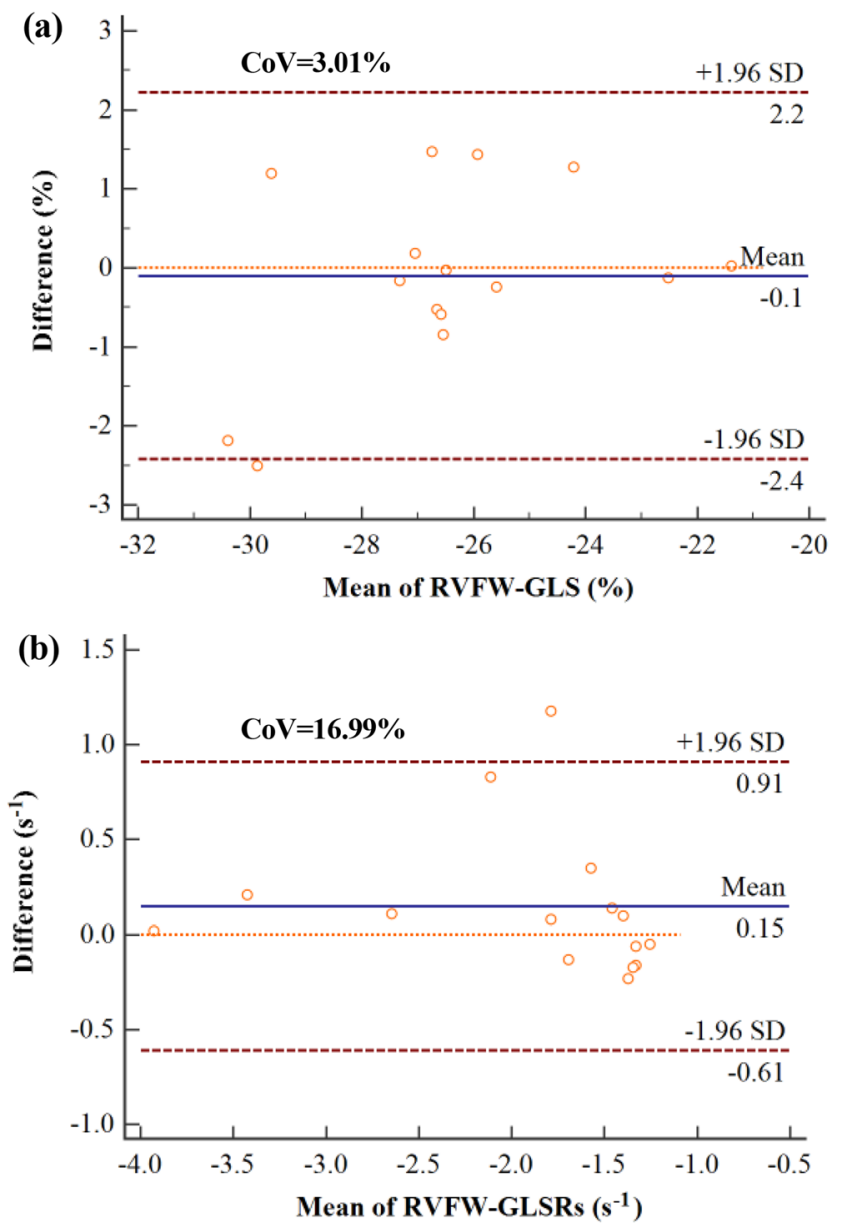

(c)

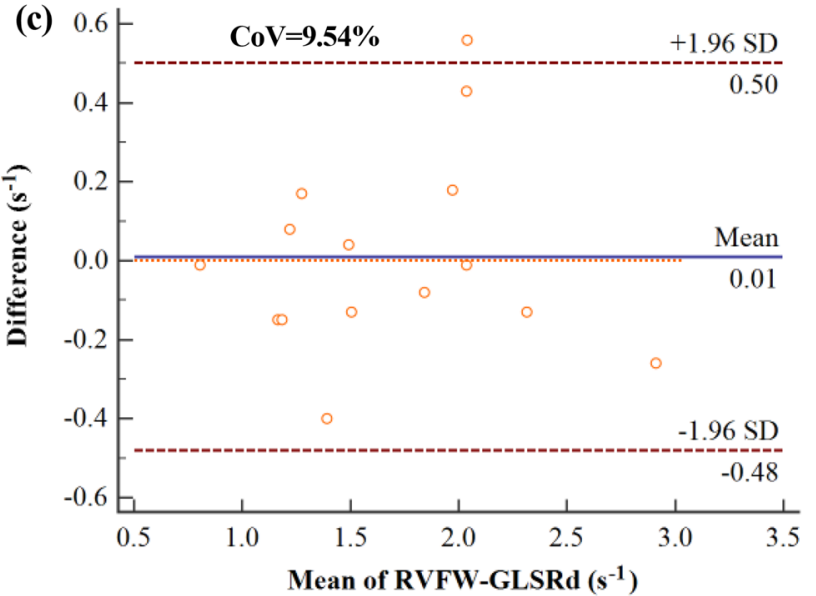

Inter-observer variability
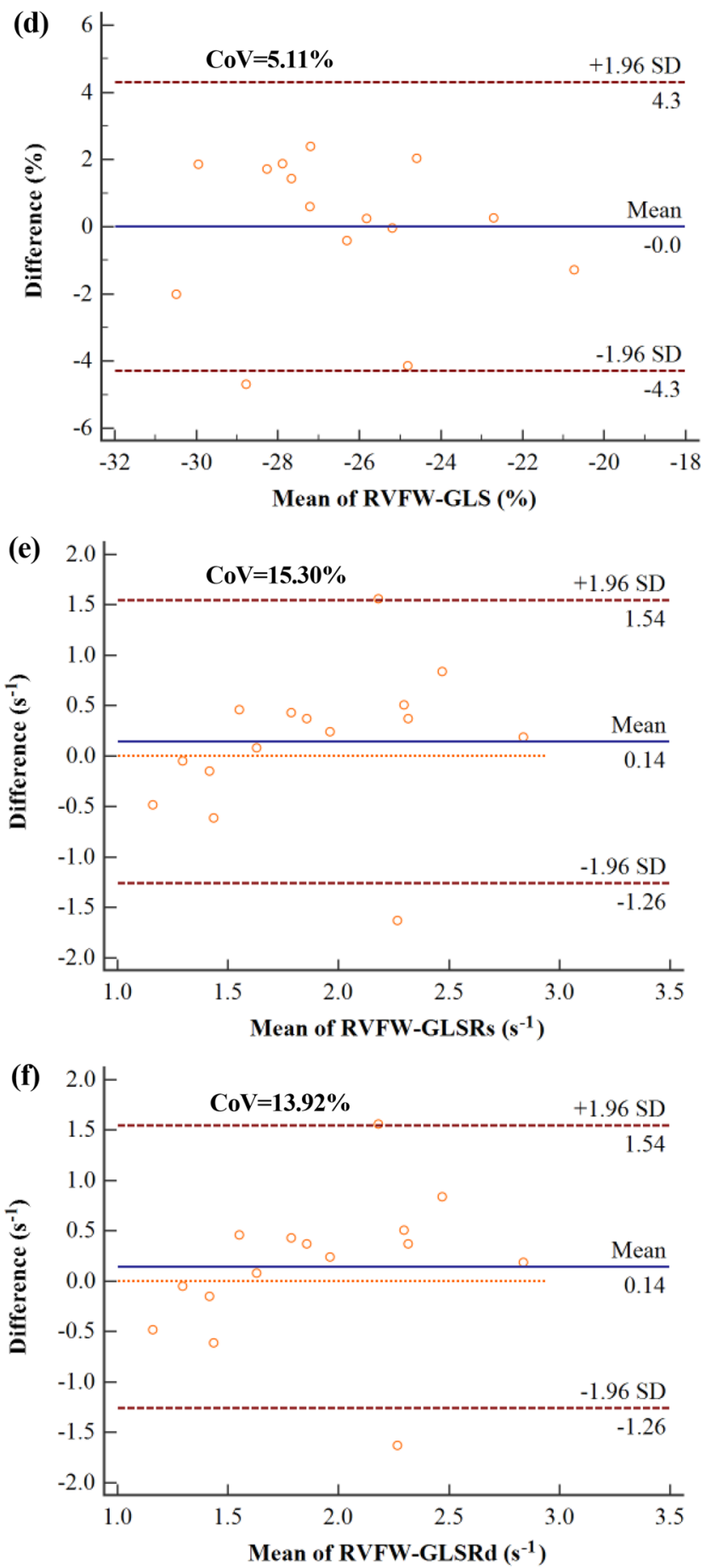

Fig. 3 Bland-Altman plots showing intra- and inter-observer variability of RVFW-GLS and RVFW-GLSR

segmental LS was no more than $11.52 \%$ except for the most apical segment $6(\mathrm{CoV}=18.24 \%$ and $29.65 \%$ for intra- and inter-observer assessment, respectively). The variability for SLSR was in the range of $10.41-29.61 \%$ and $15.11-31.83 \%$ for intra- and inter-observer assessment, respectively.

\section{Discussion}

This study investigated RVFW-LS and systolic and diastolic LSR at global, regional and segmental levels with CMRTT in 150 healthy volunteers without cardiovascular and 
Table 5 Intra- and interobserver variability of RVFW-LS

\begin{tabular}{|c|c|c|c|c|c|c|}
\hline & \multicolumn{3}{|c|}{ Intra-observer variability } & \multicolumn{3}{|c|}{ Inter-observer variability } \\
\hline & $\mathrm{MD} \pm \mathrm{SD}$ & ICC (95\% CI) & $\mathrm{CoV}$ & $\mathrm{MD} \pm \mathrm{SD}$ & ICC $(95 \%$ CI $)$ & $\mathrm{CoV}$ \\
\hline RVFW-GLS & $0.85 \pm 0.80$ & $0.94(0.83-0.98)$ & 3.01 & $1.66 \pm 1.35$ & $0.83(0.48-0.94)$ & 5.11 \\
\hline \multicolumn{7}{|l|}{ RVFW-RLS } \\
\hline Basal & $1.82 \pm 1.74$ & $0.89(0.67-0.96)$ & 5.88 & $1.92 \pm 1.46$ & $0.90(0.71-0.97)$ & 4.88 \\
\hline Middle-cavity & $1.64 \pm 1.32$ & $0.95(0.84-0.98)$ & 4.89 & $1.75 \pm 1.95$ & $0.94(0.82-0.98)$ & 7.18 \\
\hline Apical & $2.10 \pm 1.37$ & $0.84(0.54-0.95)$ & 7.43 & $2.89 \pm 1.90$ & $0.72(0.17-0.91)$ & 10.45 \\
\hline \multicolumn{7}{|l|}{ RVFW-SLS } \\
\hline Segment 1 & $2.48 \pm 2.35$ & $0.90(0.71-0.97)$ & 8.01 & $2.24 \pm 1.39$ & $0.93(0.80-0.98)$ & 4.66 \\
\hline Segment 2 & $1.48 \pm 1.65$ & $0.87(0.60-0.96)$ & 5.55 & $1.93 \pm 1.88$ & $0.84(0.52-0.95)$ & 6.28 \\
\hline Segment 3 & $1.34 \pm 0.86$ & $0.97(0.90-0.99)$ & 3.00 & $1.72 \pm 1.62$ & $0.95(0.84-0.98)$ & 5.57 \\
\hline Segment 4 & $2.48 \pm 1.89$ & $0.90(0.71-0.97)$ & 7.53 & $2.61 \pm 2.69$ & $0.88(0.66-0.96)$ & 10.70 \\
\hline Segment 5 & $1.66 \pm 1.20$ & $0.90(0.69-0.97)$ & 5.12 & $2.73 \pm 2.67$ & $0.55(-0.35-0.85)$ & 11.52 \\
\hline Segment 6 & $2.95 \pm 2.46$ & $0.83(0.50-0.94)$ & 18.24 & $4.09 \pm 3.88$ & $0.66(-0.01-0.89)$ & 29.65 \\
\hline
\end{tabular}

$M D$ mean difference, $S D$ standard deviation, $I C C$ intra-class correlation coefficient, $C I$ confidence interval, $\mathrm{CoV}$ coefficient of variation. The unit of $\mathrm{CoV}$ is \%

\begin{tabular}{|c|c|c|c|c|c|c|}
\hline & \multicolumn{3}{|c|}{ Intra-observer variability } & \multicolumn{3}{|c|}{ Inter-observer variability } \\
\hline & $\mathrm{MD} \pm \mathrm{SD}$ & ICC (95\% CI) & $\mathrm{CoV}$ & $\mathrm{MD} \pm \mathrm{SD}$ & $\mathrm{ICC}(95 \% \mathrm{CI})$ & $\mathrm{CoV}$ \\
\hline RVFW-GLSRs & $0.25 \pm 0.32$ & $0.94(0.83-0.98)$ & 16.99 & $0.30 \pm 0.28$ & $0.94(0.82-0.98)$ & 15.30 \\
\hline \multicolumn{7}{|l|}{ RVFW-RLSRs } \\
\hline Basal & $0.36 \pm 0.50$ & $0.94(0.82-0.98)$ & 15.65 & $0.70 \pm 0.59$ & $0.75(0.26-0.92)$ & 19.13 \\
\hline Middle-cavity & $0.37 \pm 0.31$ & $0.92(0.76-0.97)$ & 11.47 & $0.41 \pm 0.38$ & $0.92(0.75-0.97)$ & 13.98 \\
\hline Apical & $0.35 \pm 0.39$ & $0.91(0.74-0.97)$ & 18.62 & $0.49 \pm 0.35$ & $0.83(0.49-0.94)$ & 16.13 \\
\hline \multicolumn{7}{|l|}{ RVFW-SLSRs } \\
\hline Segment 1 & $0.65 \pm 0.76$ & $0.91(0.74-0.97)$ & 20.96 & $1.07 \pm 0.96$ & $0.62(-0.12-0.87)$ & 27.91 \\
\hline Segment 2 & $0.48 \pm 0.51$ & $0.92(0.76-0.97)$ & 18.06 & $0.72 \pm 0.54$ & $0.79(0.37-0.93)$ & 19.99 \\
\hline Segment 3 & $0.54 \pm 0.46$ & $0.87(0.61-0.96)$ & 16.76 & $0.56 \pm 0.49$ & $0.90(0.69-0.97)$ & 17.24 \\
\hline Segment 4 & $0.40 \pm 0.42$ & $0.89(0.67-0.97)$ & 15.85 & $0.47 \pm 0.42$ & $0.89(0.67-0.97)$ & 15.78 \\
\hline Segment 5 & $0.53 \pm 0.56$ & $0.83(0.49-0.94)$ & 22.91 & $0.80 \pm 0.70$ & $0.61(-0.15-0.87)$ & 27.99 \\
\hline Segment 6 & $0.44 \pm 0.36$ & $0.79(0.33-0.93)$ & 23.31 & $0.58 \pm 0.39$ & $0.62(-0.17-0.88)$ & 23.28 \\
\hline
\end{tabular}

GLSRs global longitudinal systolic peak strain rate, RLSRs regional longitudinal systolic peak strain rate, SLSRs segmental longitudinal systolic peak strain rate, $M D$ mean difference, $S D$ standard deviation, ICC intra-class correlation coefficient, $C I$ confidence interval, $C o V$ coefficient of variation. The unit of $\mathrm{CoV}$ is $\%$ pulmonary diseases. The major findings of this study are as follows: (i) CMR-TT reproducibly provides RVFW-LS and LSR by semi-automatic tracking of RV myocardial tissue features over the cardiac cycle, (ii) females presented with significantly higher amplitude of RVFW-LS and diastolic LSR than males, especially in the elderly group, (iii) even though neither an age-related difference nor a correlation between age and RVFW-GLS was observed, diastolic RVFW-GLSR in general showed a trend to decrease in males with ageing, and (iv) a base-to-apex gradient in RV longitudinal shortening was observed with almost similar contraction in basal and middle location and minimum contraction at the apical segment.

\section{The measurement of RVFW-LS and LSR with CMR-TT}

Recent improvements in cardiac imaging have made the measurement of RV function and deformation feasible and practical in clinical routine. It has proven to be useful for the early detection of RV functional and mechanical abnormalities before obvious changes in the EF. However, conventional echocardiography, as the most available and cheapest imaging modality for evaluating RV structure and function, is limited due to the insufficient image quality (narrow acoustic windows into RV) and subjective quantification $[7,22]$. Overcoming the limitations of echocardiography, CMR has emerged as the gold standard due to its high spatial 
Table 7 Intra- and interobserver variability of diastolic RVFW-LSR

\begin{tabular}{|c|c|c|c|c|c|c|}
\hline & \multicolumn{3}{|c|}{ Intra-observer variability } & \multicolumn{3}{|c|}{ Inter-observer variability } \\
\hline & $\mathrm{MD} \pm \mathrm{SD}$ & ICC (95\% CI) & $\mathrm{CoV}$ & $\mathrm{MD} \pm \mathrm{SD}$ & ICC (95\% CI) & $\mathrm{CoV}$ \\
\hline RVFW-GLSRd & $0.18 \pm 0.16$ & $0.95(0.84-0.98)$ & 9.54 & $0.22 \pm 0.23$ & $0.91(0.73-0.97)$ & 13.92 \\
\hline \multicolumn{7}{|l|}{ RVFW-RLSRd } \\
\hline Basal & $0.44 \pm 0.35$ & $0.91(0.72-0.97)$ & 10.62 & $0.54 \pm 0.39$ & $0.75(0.26-0.92)$ & 11.70 \\
\hline Middle-cavity & $0.38 \pm 0.24$ & $0.79(0.36-0.93)$ & 10.64 & $0.38 \pm 0.41$ & $0.92(0.75-0.97)$ & 18.18 \\
\hline Apical & $0.38 \pm 0.30$ & $0.85(0.54-0.95)$ & 16.07 & $0.53 \pm 0.48$ & $0.83(0.49-0.94)$ & 25.39 \\
\hline \multicolumn{7}{|l|}{ RVFW-SLSRd } \\
\hline Segment 1 & $0.72 \pm 0.68$ & $0.92(0.75-0.97)$ & 15.86 & $0.85 \pm 0.62$ & $0.92(0.77-0.97)$ & 15.11 \\
\hline Segment 2 & $0.34 \pm 0.25$ & $0.88(0.63-0.96)$ & 10.41 & $0.53 \pm 0.48$ & $0.79(0.36-0.93)$ & 19.00 \\
\hline Segment 3 & $0.51 \pm 0.58$ & $0.48(-0.54-0.83)$ & 23.72 & $0.53 \pm 0.53$ & $0.52(-0.42-0.84)$ & 22.89 \\
\hline Segment 4 & $0.60 \pm 0.36$ & $0.65(-0.04-0.88)$ & 17.32 & $0.59 \pm 0.47$ & $0.68(0.04-0.89)$ & 21.12 \\
\hline Segment 5 & $0.40 \pm 0.38$ & $0.89(0.67-0.96)$ & 17.40 & $0.71 \pm 0.54$ & $0.45(-0.63-0.82)$ & 24.55 \\
\hline Segment 6 & $0.48 \pm 0.47$ & $0.68(0.06-0.89)$ & 29.61 & $0.54 \pm 0.51$ & $0.56(-0.30-0.85)$ & 31.83 \\
\hline
\end{tabular}

GLSRd global longitudinal diastolic peak strain rate, $R L S R d$ regional longitudinal diastolic peak strain rate, $S L S R d$ segmental longitudinal diastolic peak strain rate, $M D$ mean difference, $S D$ standard deviation, ICC intra-class correlation coefficient, $\mathrm{CI}$ confidence interval, $\mathrm{CoV}$ coefficient of variation. The unit of $\mathrm{CoV}$ is $\%$ and temporal resolution, good soft tissue contrast without ionizing radiation.

CMR-TT has been developed to quantify dynamic deformation of the myocardium. CMR-TT is based on conventional SSFP cine images and does not require any additional measurements as in tissue tagging [11]. Deformation information is derived from automatic tracking of tissue features over the cardiac cycle [13] and has presented as sensitive and convenient offline post-processing analysis [23].

Myocardial strain is defined as the relative lengthening and thickening (known as positive strain) or shortening and thinning (known as negative strain) of myocardial fibers compared with the end-diastolic length. Strain is the most frequently used index to estimate myocardial deformation. The strain rate (SR), represented as the derivative of the strain, quantifies the degree of change in myocardial deformation with respect to time [13]. Strain and SR can be applied to analyze myocardial deformation at global and segmental levels. Both parameters have been reported to be relatively independent on loading conditions and to identify active contraction [24], and as such to be advantageous in comparison to conventional volumetric parameters such as EF and stroke volume.

The longitudinal shortening is the major contribution to RV contraction, and RV function was shown highly dependent on longitudinal shortening [25]. LS and LSR may identify subclinical RV dysfunction before the development of abnormalities reflected by RVEF or stroke volume (as similar as in LV) [11]. Considering that interventricular septum is mainly a constituent part of the LV and only contributes $20 \%$ to RV systolic performance [26], analysis of the RVFW appears superior to quantify the RV contraction.
RVFW-GLS was reported to be the most accurate functional marker correlating with the degree of RV myocardial fibrosis in patients with advanced HF [26]. It also been shown as a prognostic marker in acute non-massive pulmonary embolism with area under the curve (AUC) as 0.754 [27]. In addition, intraoperative RV functional decline was found to be primarily related with deterioration of RVFW deformation rather than that of the interventricular septum [28]. Further RVFW-GLS has been reported to provide important diagnostic information in patients with depressed RVEF (AUC=0.918) [29], RV pressure overload $(\mathrm{AUC}=0.95)$ [30], and proximal right coronary artery lesion-induced ischemic heart disease $(\mathrm{AUC}=0.79)$ [31].

Even though RVFW-LS and LSR have indicated to be clinical relevant parameters, age- and gender-specific reference values for global an segmental analysis are rare. In this contribution, respective reference values for CMR-TT were derived from four-chamber long-axis SSFP cine images with a strong focus on the free wall.

\section{Reproducibility of RVFW-LS and LSR}

Liu et al. derived a mean RVFW-GLS of $-24.2 \pm 3.59 \%$ among 100 normal healthy subjects, which was in good concordance with our result of $-24.9 \pm 5.1 \%$. The systolic RVFW-GLSR was as $-1.54 \pm 0.41 \mathrm{~s}^{-1}$, which was close to the data we obtained [19]. The RVFW is thinner and thus more potential to present higher longitudinal deformation against pulmonary resistance compared to the interventricular septum [32]. Hence the reference values of RVFW-GLS derived from the RVFW only couldn't be simply utilized to estimate the mechanical deformation of the whole RV 
myocardium. Further, the reference values aren't interchangeable for other modalities or vendors using different algorithms. For example, the average value of RVFW-LS acquired by Mararu et al. using 2-dimensional speckletracking echocardiography was $-30.5 \pm 3.9 \%$, which was far from ours [33].

It's a consensus that strain measurement is more reliable than SR, and global evaluation is superior to segmental assessment. This observation was confirmed by our intraand inter-observer reproducibility assessment. The poorest reproducibility of SLS and SLSR in apical segments may limit the regional application of CMR-TT technique in measuring RVFW apical deformation. The lower intra- and inter-observer variability of RVFW-GLS found in ours and others' researches $[21,34]$ clearly indicate the use of the global parameter as a reliable and reproducible clinical and research parameter.

\section{Gender- and age-related differences of RVFW-LS and LSR}

Gender-related differences of RVFW-LS and LSR have been discussed before. Truong et al. analyzed the RVFW-GLS and SLS among 50 patients (4-81 years old) without known cardiac pathology with the same software package. He demonstrated that the GLS was comparable between males and females as $-22.22 \pm 3.4 \%$ and $-22.80 \pm 3.5 \%$, respectively [22]. Liu et al. also reported no significant differences with values of $-23.9 \pm 3.59 \%$ for males and $-24.6 \pm 3.59 \%$ for females $(P=0.34)$ [35]. However, our study reports a significant higher amplitude of RVFW-GLS in females compared with that in males as $-26.1 \pm 5.2 \%$ vs. $-23.7 \pm 4.9 \%$ $(P<0.05)$ on the basis of a larger sample size. Our finding is consistent with previous studies including the largest echocardiographic speckle-tracking study performed among 276 healthy volunteer by Muraru et al. [22, 33]. We hypothesized that the gender-related difference of GLS was probably caused by the different RV size and systolic function, since males presented with higher RVEDVI, RVESVI and RESVI, and lower RVEF. The systolic LSR was comparable between males and females $(P>0.05)$, which implies the similar rate of myocardial shortening during systole. The RVFW diastolic LSR is regarded as a marker of RV relaxation, which may reduce with collagen deposition, fibrosis and impaired calcium uptake of cardiomyocytes [36]. Females presented higher diastolic GLSR compared with males as $1.80 \pm 0.67 \%$ vs. $1.59 \pm 0.62 \%(P<0.05)$, implying the better compliance of RVFW. Meanwhile, the decrease of diastolic GLSR in males demonstrated the deterioration of diastolic function of RVFW with ageing. In contrast, the absence of a significant decrease in the diastolic LSR in females might be explained by the low feasibility of SR assessment.
With the potential age-related increase in pulmonary artery pressure, pulmonary vascular resistance, cardiomyocyte loss and subsequent replacement by fibrosis, RVFW mechanical function was supposed to decrease with aging [36]. We did not find any age-related difference of RVFWGLS and systolic GLSR in neither gender groups and no correlation between age and RVFW-GLS or systolic GLSR. This finding was in line with the finding of previous studies $[22,33,37]$. This finding may simplify the establishment of reference values and clinical use of these two parameters. However, normative data from multicenter and larger size of population are still necessary for the further validation.

\section{Segmental variability of RVFW-SLS and SLSR}

The RVFW base-to-apex gradient was measured and resulted as $-27.1 \pm 7.2 \%,-27.2 \pm 4.9 \%$ and $-19.8 \pm 6.6 \%$ for SLS, and $-2.84 \pm 0.95 \%,-2.79 \pm 0.90 \%$ and $-2.08 \pm 0.70 \%$ for systolic SLSR, respectively $(P<0.05)$. RVFW-SLS and systolic SLSR reached the lowest values in the apical territories and became comparable between the basal and mid-cavity regions. Our outcome is in good accordance the finding from a meta-analysis of 226 healthy children $(<21$ years of age) from 10 studies [38] and others' researches [32, 39]. Abnormality of this physiological principle of base-to-apex heterogeneity may differentiate the early alteration of RV myocardial contractility or afterload among patients with various pathological changes. Furthermore, even though the reproducibility of segmental strain and SR are lower than global parameters [40], the awareness of segmental reference values and alterations contributes to the better understanding and localization of impaired myocardium.

\section{Study limitation}

There were several limitation to this study. Firstly, this is a single-center study among a limited number $(n=150)$ of healthy volunteers. Even though the number of healthy subjects enrolled in this study exceeds most previous studies, investigations in multiple centers with larger sample size are still necessary to further clarify the intrinsic principle of RVFW-LS and LSR. Secondly, it's challenging to quantify $\mathrm{RV}$ myocardial deformation due to the thin wall and crescent shape. Hence, we merely focused on the deformation in the longitudinal direction with long-axis four-chamber view cine images, which theoretically ensured the good feasibility and reproducibility. Manual adjustment and visual inspection by an experienced physicians were performed during the measurement, which ensured the quality of operation. Thirdly, the reference values may be vendor-specific and likely do not apply with other vendors. 


\section{Conclusion}

In conclusion, our study provides reference values of RVFW-LS and LSR for specific vendor $\mathrm{CVI}^{42}$ using CMRTT technique. Meanwhile, the gender- and age-related differences and base-to-apex gradient of RVFW-LS and LSR were investigated among a relatively large size of healthy population. The vendor-, gender- and segment-specific reference values we established with CMR-TT modality contribute to the standard incorporation of RVFW global and segmental LS and LSR into the clinical routine.

Acknowledgments Open Access funding provided by Projekt DEAL.

Author contributions VR was responsible for the design and overall investigation. DB was responsible of recruiting healthy volunteers. YYQ was responsible for the measurement, data collection, statistical analysis and manuscript. HL helped complete the inter-observer reproducibility assessment. VR, YYQ, DB, WR and GSM have made substantial contributions to analysis and interpretation of data, drafting or revising the manuscript. All authors read and approved the final manuscript.

Data availability The datasets used and/or analyzed during the current study are available from the corresponding author on reasonable request.

\section{Compliance with ethical standards}

Conflict of interest The authors declare that they have no competing interest.

Ethical approval and consent to participate The study was approved by the local ethics committee. All subjects supplied written informed consent.

Open Access This article is licensed under a Creative Commons Attribution 4.0 International License, which permits use, sharing, adaptation, distribution and reproduction in any medium or format, as long as you give appropriate credit to the original author(s) and the source, provide a link to the Creative Commons licence, and indicate if changes were made. The images or other third party material in this article are included in the article's Creative Commons licence, unless indicated otherwise in a credit line to the material. If material is not included in the article's Creative Commons licence and your intended use is not permitted by statutory regulation or exceeds the permitted use, you will need to obtain permission directly from the copyright holder. To view a copy of this licence, visit http://creativecommons.org/licenses/by/4.0/.

\section{References}

1. Haddad F, Doyle R, Murphy DJ, Hunt SA (2008) Right ventricular function in cardiovascular disease, part II: pathophysiology, clinical importance, and management of right ventricular failure. Circulation 117(13):1717-1731

2. Sanz J, Sanchez-Quintana D, Bossone E, Bogaard HJ, Naeije R (2019) Anatomy, function, and dysfunction of the right ventricle: JACC state-of-the-art review. J Am Coll Cardiol 73(12):1463-1482

3. Voelkel NF, Quaife RA, Leinwand LA, Barst RJ, McGoon MD, Meldrum DR, Dupuis J, Long CS, Rubin LJ, Smart FW, Suzuki YJ, Gladwin M, Denholm EM, Gail DB, National Heart L, Blood Institute Working Group on C, Molecular Mechanisms of Right Heart F (2006) Right ventricular function and failure: report of a National Heart, Lung, and Blood Institute working group on cellular and molecular mechanisms of right heart failure. Circulation 114(17): 1891

4. Prasad SK, Lota AS (2017) Right ventricle dysfunction in cardiomyopathy: to measure is to know. JACC Cardiovasc Imaging 10(10):1237-1239

5. Sabe MA, Sabe SA, Kusunose K, Flamm SD, Griffin BP, Kwon DH (2016) Predictors and prognostic significance of right ventricular ejection fraction in patients with ischemic cardiomyopathy. Circulation 134(9):656-665

6. Klinke V, Muzzarelli S, Lauriers N, Locca D, Vincenti G, Monney P, Lu C, Nothnagel D, Pilz G, Lombardi M, van Rossum AC, Wagner A, Bruder O, Mahrholdt H, Schwitter J (2013) Quality assessment of cardiovascular magnetic resonance in the setting of the European CMR registry: description and validation of standardized criteria. J Cardiovasc Magn Reson 15:55

7. Suever JD, Wehner GJ, Jing LY, Powell DK, Hamlet SM, Grabau JD, Mojsejenko D, Andres KN, Haggerty CM, Fornwalt BK (2017) Right ventricular strain, torsion, and dyssynchrony in healthy subjects using 3D spiral cine DENSE magnetic resonance imaging. IEEE Trans Med Imaging 36(5):1076-1085

8. Mouton S, Ridon H, Fertin M, Pentiah AD, Goemine C, Petyt G, Lamblin N, Coisne A, Foucher-Hossein C, Montaigne D, de Groote P (2017) 2D-speckle tracking right ventricular strain to assess right ventricular systolic function in systolic heart failure. Analysis of the right ventricular free and posterolateral walls. Int J Cardiol 245:190-195

9. Park JH, Negishi K, Kwon DH, Popovic ZB, Grimm RA, Marwick TH (2014) Validation of global longitudinal strain and strain rate as reliable markers of right ventricular dysfunction: comparison with cardiac magnetic resonance and outcome. J Cardiovasc Ultrasound 22(3):113-120

10. Hu BY, Wang J, Yang ZG, Ren Y, Jiang L, Xie LJ, Liu X, Gao Y, Shen MT, Xu HY, Shi K, Li ZL, Xia CC, Peng WL, Deng MY, Li H, Guo YK (2019) Cardiac magnetic resonance feature tracking for quantifying right ventricular deformation in type 2 diabetes mellitus patients. Sci Rep 9(1):11148

11. de Siqueira MEM, Pozo E, Fernandes VR, Sengupta PP, Modesto K, Gupta SS, Barbeito-Caamano C, Narula J, Fuster V, Caixeta A, Sanz J (2016) Characterization and clinical significance of right ventricular mechanics in pulmonary hypertension evaluated with cardiovascular magnetic resonance feature tracking. J Cardiovasc Magn Reson 18(1):39

12. Tello K, Dalmer A, Vanderpool R, Ghofrani HA, Naeije R, Roller F, Seeger W, Wilhelm J, Gall H, Richter MJ (2019) Cardiac magnetic resonance imaging-based right ventricular strain analysis for assessment of coupling and diastolic function in pulmonary hypertension. JACC Cardiovasc Imaging 12(11 Pt 1):2155-2164

13. Pedrizzetti G, Claus P, Kilner PJ, Nagel E (2016) Principles of cardiovascular magnetic resonance feature tracking and echocardiographic speckle tracking for informed clinical use. J Cardiovasc Magn Reson 18(1):51

14. Schuster A, Stahnke VC, Unterberg-Buchwald C, Kowallick JT, Lamata P, Steinmetz M, Kutty S, Fasshauer M, Staab W, Sohns JM, Bigalke B, Ritter C, Hasenfuß G, Beerbaum P, Lotz J (2015) Cardiovascular magnetic resonance feature-tracking assessment of myocardial mechanics: intervendor agreement and considerations regarding reproducibility. Clin Radiol 70(9):989-998 
15. Hwang JW, Kim SM, Park SJ, Cho EJ, Kim EK, Chang SA, Lee SC, Choe YH, Park SW (2017) Assessment of reverse remodeling predicted by myocardial deformation on tissue tracking in patients with severe aortic stenosis: a cardiovascular magnetic resonance imaging study. J Cardiovasc Magn Reson 19(1):80

16. Muser D, Tioni C, Shah R, Selvanayagam JB, Nucifora G (2017) Prevalence, correlates, and prognostic relevance of myocardial mechanical dispersion as assessed by feature-tracking cardiac magnetic resonance after a first ST-segment elevation myocardial infarction. Am J Cardiol 120(4):527-533

17. Taylor RJ, Moody WE, Umar F, Edwards NC, Taylor TJ, Stegemann B, Townend JN, Hor KN, Steeds RP, Mazur W, Leyva F (2015) Myocardial strain measurement with feature-tracking cardiovascular magnetic resonance: normal values. Eur Heart J Cardiovasc Imaging 16(8):871-881

18. Yingchoncharoen T, Agarwal S, Popovic ZB, Marwick TH (2013) Normal ranges of left ventricular strain: a meta-analysis. J Am Soc Echocardiogr 26(2):185-191

19. Liu BY, Dardeer AM, Moody WE, Edwards NC, Hudsmith LE, Steeds RP (2018) Normal values for myocardial deformation within the right heart measured by feature-tracking cardiovascular magnetic resonance imaging. Int J Cardiol 252:220-223

20. Stiermaier T, Lange T, Chiribiri A, Moller C, Graf T, Raaz U, Villa A, Kowallick JT, Lotz J, Hasenfuss G, Thiele H, Schuster A, Eitel I (2018) Right ventricular strain assessment by cardiovascular magnetic resonance myocardial feature tracking allows optimized risk stratification in Takotsubo syndrome. PLoS ONE 13(8): 0202146

21. Liu T, Wang C, Li S, Zhao Y, Li P (2019) Age- and gender-related normal references of right ventricular strain values by tissue tracking cardiac magnetic resonance: results from a Chinese population. Quant Imaging Med Surg 9(8):1441-1450

22. Truong VT, Safdar KS, Kalra DK, Gao X, Ambach S, Taylor MD, Moore R, Taylor RJ, Germann J, Toro-Salazar O, Jefferies JL, Bartone C, Raman SV, Ngo T, Mazur W (2017) Cardiac magnetic resonance tissue tracking in right ventricle: feasibility and normal values. Magn Reson Imaging 38:189-195

23. Scatteia A, Baritussio A, Bucciarelli-Ducci C (2017) Strain imaging using cardiac magnetic resonance. Heart Fail Rev 22(4):465-476

24. Visentin S, Palermo C, Camerin M, Daliento L, Muraru D, Cosmi E, Badano LP (2017) Echocardiographic techniques of deformation imaging in the evaluation of maternal cardiovascular system in patients with complicated pregnancies. Biomed Res Int 2017:4139635

25. Carlsson M, Ugander M, Heiberg E, Arheden H (2007) The quantitative relationship between longitudinal and radial function in left, right, and total heart pumping in humans. Am J Physiol-Heart C 293(1):H636-H644

26. Sanz-de la Garza M, Giraldeau G, Marin J, Imre Sarvari S, Guasch E, Gabrielli L, Brambila C, Bijnens B, Sitges M (2019) Should the septum be included in the assessment of right ventricular longitudinal strain? An ultrasound two-dimensional speckle-tracking stress study. Int J Cardiovasc Imaging 35(10):1853-1860

27. Lee K, Kwon O, Lee EJ, Sin MJ, Lee JS, Lee S, Kang DH, Song JK, Song JM (2019) Prognostic value of echocardiographic parameters for right ventricular function in patients with acute nonmassive pulmonary embolism. Heart Vessels 34(7):1187-1195

28. Rong LQ, Yum B, Abouzeid C, Palumbo MC, Brouwer LR, Devereux RB, Girardi LN, Weinsaft JW, Gaudino M, Kim J (2019) Echocardiographic predictors of intraoperative right ventricular dysfunction: a 2D and speckle tracking echocardiography study. Cardiovasc Ultrasound 17(1):11
29. Focardi M, Cameli M, Carbone SF, Massoni A, De Vito R, Lisi M, Mondillo S (2015) Traditional and innovative echocardiographic parameters for the analysis of right ventricular performance in comparison with cardiac magnetic resonance. Eur Heart J Cardiovasc Imaging 16(1):47-52

30. Hardegree EL, Sachdev A, Villarraga HR, Frantz RP, McGoon MD, Kushwaha SS, Hsiao JF, McCully RB, Oh JK, Pellikka PA, Kane GC (2013) Role of serial quantitative assessment of right ventricular function by strain in pulmonary arterial hypertension. Am J Cardiol 111(1):143-148

31. Chang WT, Tsai WC, Liu YW, Lee CH, Liu PY, Chen JY, Li YH, Tsai LM (2014) Changes in right ventricular free wall strain in patients with coronary artery disease involving the right coronary artery. J Am Soc Echocardiogr 27(3):230-238

32. Rimbas RC, Mihaila S, Enescu OA, Vinereanu D (2016) A new comprehensive 12-segment approach to right ventricular systolic and diastolic functions by 2D speckle tracking echocardiography in healthy individuals. Echocardiography 33(12):1866-1873

33. Muraru D, Onciul S, Peluso D, Soriani N, Cucchini U, Aruta P, Romeo G, Cavalli G, Iliceto S, Badano LP (2016) Sex- and method-specific reference values for right ventricular strain by 2-dimensional speckle-tracking echocardiography. Circ Cardiovasc Imaging 9(2):e003866

34. Peng J, Zhao X, Zhao L, Fan Z, Wang Z, Chen H, Leng S, Allen J, Tan RS, Koh AS, Ma X, Lou M, Zhong L (2018) Normal values of myocardial deformation assessed by cardiovascular magnetic resonance feature tracking in a healthy Chinese population: a multicenter study. Front Physiol 9:1181

35. Liu B, Dardeer AM, Moody WE, Edwards NC, Hudsmith LE, Steeds RP (2018) Reference ranges and reproducibility studies for right heart myocardial deformation by feature tracking cardiovascular magnetic resonance imaging. Data Brief 16:244-249

36. Chia EM, Hsieh CHC, Boyd A, Pham P, Vidaic J, Leung D, Thomas L (2014) Effects of age and gender on right ventricular systolic and diastolic function using two-dimensional speckletracking strain. J Am Soc Echocardiogr 27(10):1079-U1175

37. Salehi Ravesh M, Rickers C, Bannert FJ, Hautemann D, Al Bulushi A, Gabbert DD, Wegner P, Kis E, Hansen JH, JeroschHerold M, Kramer HH, Logoteta J (2018) Longitudinal deformation of the right ventricle in hypoplastic left heart syndrome: a comparative study of $2 \mathrm{D}$-feature tracking magnetic resonance imaging and 2D-speckle tracking echocardiography. Pediatr Cardiol 39(6): 1265-1275

38. Levy PT, Mejia AAS, Machefsky A, Fowler S, Holland MR, Singh GK (2014) Normal ranges of right ventricular systolic and diastolic strain measures in children: a systematic review and meta-analysis. J Am Soc Echocardiogr 27(5):549-U145

39. Kemal HS, Kayikcioglu M, Kultursay H, Vuran O, Nalbantgil S, Mogulkoc N, Can L (2017) Right ventricular free-wall longitudinal speckle tracking strain in patients with pulmonary arterial hypertension under specific treatment. Echocardiography 34(4):530-536

40. Mirea O, Berceanu M, Donoiu I, Militaru C, Saftoiu A, Istratoaie $\mathrm{O}$ (2019) Variability of right ventricular global and segmental longitudinal strain measurements. Echocardiography 36(1):102-109

Publisher's Note Springer Nature remains neutral with regard to jurisdictional claims in published maps and institutional affiliations. 